\title{
Autosomal recessive intermediate Charcot-Marie-Tooth disease type B
}

INSERM

\section{Source}

INSERM. (1999). Orphanet: an online rare disease and orphan drug data base. Autosomal recessive intermediate Charcot-Marie-Tooth disease type B. ORPHA:254334

Autosomal recessive intermediate Charcot-Marie-T ooth disease type $B$ is an extremely rare subtype of autosomal recessive intermediate Charcot-Marie-Tooth (CMT) disease characterized by a CMT neuropathy associated with developmental delay, self-abusive behavior, dysmorphic features and vestibular Schwannoma. Motor nerve conduction velocities demonstrate features of both demyelinating and axonal pathology. 